\title{
Pathways from dieting to weight regain, to obesity and to the metabolic syndrome: an overview
}

\author{
A. G. Dulloo and J.-P. Montani
}

\author{
Department of Medicine, Division of \\ Physiology, University of Fribourg, Fribourg, \\ Switzerland
}

Address for correspondence: AG Dulloo, Department of Medicine/Physiology, University of Fribourg, Chemin du musée 5, CH-1700 Fribourg, Switzerland.

E-mail: abdul.dulloo@unifr.ch

\begin{abstract}
Summary
Every year, scores of millions of people - as diverse as obese and lean, teenagers and older adults, sedentary and elite athletes, commoners and celebrities - attempt to lose weight on some form of diet. They are often encouraged by their parents, friends, health professionals, training coaches, a media that promotes a slim image and a diet-industry that in Europe and United States alone has an annual turnover in excess of $\$ 150$ billion. Weight regain is generally the rule, with one-third to two-thirds of the weight lost being regained within 1 year and almost all is regained within 5 years. With studies of the long-term outcomes showing that at least one-third of dieters regain more weight than they lost, together with prospective studies indicating that dieting during childhood and adolescence predicts future weight gain and obesity, there is concern as to whether dieting may paradoxically be promoting exactly the opposite of what it is intended to achieve. Does dieting really make people fatter? How? Does dieting increase the risks for cardiometabolic diseases as many go through repeated cycles of intentional weight loss and unintentional weight regain, i.e. through yo-yo dieting or weight cycling? What's new in adipose tissue biology pertaining to the mechanisms that drive weight regain? Why does exercise not necessarily work in concert with dieting to achieve weight loss and prevent weight regain? What 'lessons' are we learning from bariatric surgery about the mechanisms by which long-term weight loss seems achievable? It is these questions, against a background of preoccupation with dieting, that recent advances and controversies relevant to the theme of 'Pathways from dieting to weight regain, to obesity and to the metabolic syndrome' are addressed in this overview and the eight review articles in this supplement reporting the proceedings of the 7th Fribourg Obesity Research Conference.
\end{abstract}

Keywords: Dietary restraint, dieting, obesity, weight cycling.

\section{Introduction}

The 7th Fribourg Obesity Research Conference was attended by researchers from both academia and industry and once again provided a platform for scientists in both basic science and clinical medicine to present and discuss some of the rapidly advancing scientific findings pertaining obesity and metabolic health from a perspective of integrative physiology and nutrition. Its proceedings consist of a total of eight review articles which, together with abstracts of poster sessions, are published in this supplement. An overview of the topics and main issues addressed at this conference and in the review articles is provided in the succeeding sections. 


\section{Who are the dieters? Who are at risk for cardiometabolic diseases?}

Despite its well-documented failures over the past decades, the ancient prescription of Hippocrates that the obese should 'eat less and exercise more' (1), is still today, and for the foreseeable future, the cornerstone approach in obesity therapy. While it is generally assumed that adherence to weight reduction diets is beneficial to health, the high rates of dieting and weight loss recidivism continue to raise concerns about the long-term adverse health consequences of dieting and weight cycling. Initially embodied in 'Dieting makes you fat', the title of a book published in the early 1980s (2), dieting and weight cycling have since been repeatedly implicated in increased risk for eating disorders and other psychological disorders (e.g. increased anxiety and depression), deficits in certain aspects of cognitive performance, in a variety of increased morbidities that includes type 2 diabetes, hypertension, cancer, diminished bone density and risks for bone fracture, inflammation and even increased mortality (3). However, the long-term health consequences of dieting and weight cycling are highly controversial, with ongoing debates about whether it is prudent to even recommend that overweight or obese adults should try to lose weight (4). These discrepancies could be attributed to differences in population groups under study and to differences in methodological approaches used for their assessment, including a lack of clear distinction between intentional and unintentional weight loss or between dieting to lose weight versus dietary restraint to prevent weight gain. Furthermore, the lack of a consensus for a standard definition of weight cycling makes it difficult to compare studies and hence in drawing conclusions.

Nonetheless, as emphasized in the first review paper in this issue by Montani et al. (5), an analysis of data from the literature suggests that it is weight cycling resulting from repetitive intentional weight loss in primarily normalweight population groups that seems to be more strongly associated with risks for metabolic and cardiovascular diseases. In addressing the question of 'Who are the dieters?', these authors underscore the fact that dieting and weight cycling are not limited to those who are obese or overweight but that the prevalence of dieting to lose weight is substantial (and on the rise) in normal-weight, and even underweight, population subgroups. Such weight loss dieting is shown to be prevalent in both females and males, young and older adults as well as in adolescents and even children who perceive themselves as being too fat (because of media, parental and social pressures), among athletes in weight-sensitive sports (e.g. those with mandatory weight categories, as well as in gravitational and aesthetic sports) and also among performers for whom a slim image is professionally an advantage. In the second part of their review, they bring forward the cardiometabolic risk factors that have so far been identified in normal-weight dieters and underscore the findings from rare studies of experimentally induced weight cycling which support the notion that fluctuations of cardiovascular risk variables (such as blood pressure, heart rate, sympathetic activity, blood glucose, lipids and insulin) with probable repeated overshoots above normal values during periods of weight regain put an additional stress on the cardiovascular system. They predict that, because the onset of a pattern of weight cycling is shifting towards younger ages and as cardiovascular risk factors act together in a multiplicative way to lead slowly over years to cardiovascular events, an increase in the prevalence of cardiovascular and renal diseases associated with weight cycling is expected in the next few decades.

\section{Dieting and fatness: the chicken and egg debate}

While it is now recognized that dieting in the normalweight children, adolescents and younger adults is a strong predictor of future weight gain, the proposed explanations for this association between dieting and fatness, and in particular whether or not dieting and weight cycling play a causative role for subsequent weight gain and contribute to the current obesity epidemic, is a hotly debated topic. Some have argued that it is not that dieting makes people fatter, but that an inherent predisposition to obesity and fear of becoming fatter in an obesogenic environment make lean people more likely to go on a diet (6). In addressing the question of whether dieting is a proxy or cause of future weight gain in this issue, Lowe (7) emphasizes that the act of going on weight loss diets among those who are in or near the healthy weight range may represent an early warning sign that the dieter is experiencing weight gain that he or she wants to resist or reverse. He proposes that the act of going on weight loss diets might also be a marker (and a consequence) of an already existing predisposition towards weight gain, which is known to be strongly determined by genetic, family and other environmental influences. A key message from his review is that there is little reason to believe that losing weight via dieting produces weight regain beyond that which would have been gained if a diet had never been undertaken while living in an obesogenic environment. This contention, however, rests upon an 'abstract' quantification of post-dieting weight regain versus spontaneous weight gain in the same individuals, over the same time-period and in the same environment, and hence cannot be tested experimentally. Furthermore, the argument that genetic and familial predisposition to fatness is the driving force behind dieting in the lean cannot be used to explain why elites athletes who were practising power sports (e.g. boxing, weight lifting, wrestling) - where weight cycling is common - are also predisposed to greater 
weight gain than athletes engaged in sports without weight cycling or compared with the general population (8). Indeed, a recent large-scale, prospective study in twins, while confirming that dieters may be more prone to future weight gain and that dieters have a genetic propensity for obesity, also provides strong evidence that, in an essentially normal-weight cohort, dieting per se may promote subsequent weight gain independently of genetic, familial and other environmental factors (9). How then does dieting make lean people fatter?

\section{How dieting may predispose to fatness}

One explanation may reside in preoccupations with food and food obsession which have often been described long after episodes of food deprivation or dietary restraint. Indeed, the central tenet of proponents for a causative impact of dieting on future weight gain and obesity centres upon the notion that dieting, and in particular unhealthy dieting (e.g. use of diuretics, diet pills or laxatives, vomiting), predispose to eating disorders, disinhibition and binge eating, i.e. periods of dietary restriction alternating with episodes of uncontrolled overeating. But this does not explain why it is those who are lean, rather those who are overweight or obese, seem to be most prone to the impact of dieting on subsequent weight gain. In addressing this issue, Dulloo et al. (10) point to human studies of experimental starvation and refeeding which suggest that (i) the fraction of weight lost as fat-free mass (FFM) increases with leanness, (ii) that feedback signals from depletion of both fat mass and FFM contribute to weight regain through effects on energy intake and adaptive thermogenesis, (iii) that a faster rate of fat recovery relative to FFM recovery (i.e. preferential catch-up fat) is a central outcome of body composition autoregulation in lean individuals and (iv) that such a temporal desynchronization in the restoration of the body's fat versus FFM results in a state of hyperphagia that persists beyond complete recovery of fat mass and until full FFM recovery, and as the complete recovery of FFM is also accompanied by fat deposition, excess fat accumulates leading to fat overshooting. According to these authors, these findings confer biological plausibility for post-dieting fat overshooting - which through repeated dieting and weight cycling would increase the risks for trajectories from leanness to fatness.

An implication of the above-mentioned findings is that for the same amount of weight loss, the lean dieters would show a much greater depletion of their fat mass and FFM (relative to pre-weight loss values) than the obese dieters, and would thus be at greater risk for temporal desynchronization of fat and FFM recoveries and fat overshooting. One might hence speculate that if the obese were to show large enough weight losses resulting in marked depletion of both fat and FFM, they may also show enhanced metabolic efficiency driving fat regain and fat overshooting. However, as Bosy-Westphal et al. (11) remind us, only a few studies have compared the composition of sequential weight loss and weight regain in overweight or obese subjects and the results are conflicting with some studies reporting a disproportional regain in total or abdominal fat whereas others found no adverse effect of weight cycling on obesity or body fat distribution.

\section{Deep body composition phenotyping}

In reviewing the impact of weight cycling on body composition, metabolic efficiency and metabolic risks in overweight and obese individuals, Bosy-Westphal et al. (11) point to several confounding factors, and in particular methodological drawbacks, that limit the interpretation of body composition results, and by extension, the interpretation of a role for altered metabolic efficiency and adaptive thermogenesis in weight loss or weight regain - as these are derived from changes in energy expenditure that require adjustments for changes in body composition. They discuss how the measurement as well as prediction of changes in body composition are complicated by the fact that the fraction of weight loss or weight regain as FFM that is undergoing dynamic changes over time is often confounded by hydration of FFM because of intracellular water bound to glycogen or protein or because of water associated to sodium excretion or retention. They make the case for the need to go well beyond the assessment of body composition as a two-compartment model (fat and FFM) and for deep body composition phenotyping at the organ and tissue level because metabolic risk differs between adipose tissue depots and lean mass is metabolically heterogeneous, being composed of organs and tissues differing in metabolic rate.

\section{Biology of weight regain: the 'nutrient clearance' hypothesis}

In their review focusing specifically on the adaptations in white adipose tissues that contribute to the biological drive to regain weight after weight loss, MacLean et al. (12) emphasize that the decline in the size of the adipocytes alters their metabolic characteristics in a manner that primes adipose tissues for the rapid clearance and storage of ingested energy. These adaptations for the restoration of body fat would contribute to the attenuated postprandial excursions of circulating nutrients (e.g. glucose, triglycerides and fatty acids) following weight loss. According to their 'nutrient clearance' hypothesis, the energy gap between increased appetite and diminished energy expenditure in response to weight loss would persist during weight regain as a function of adipose tissue to clear and store excess energy, and as weight regain progresses, the adipocytes gradually increase in size and their capacity to 
clear excess energy diminishes. Once the adipocytes reach a critical threshold of size and the maximal capacity for stored energy is approached, the rate of weight regain would diminish. As these authors elaborate in their review, this 'nutrient clearance' hypothesis integrates several important features of the biology of weight regain from an adipocentric standpoint. First, it integrates the long-term adiposity signals such as leptin and insulin (which reflect the level of stored energy rather than dynamic changes in fat content of adipose tissue) with short-term signals of nutrient availability, which essentially reflect the capacity to store energy. Second, it raises the possibility that the integrated feedback signal from adipose tissues includes feedback from multiple adipose depots that have different metabolic and cellularity characteristics. Third, it can also incorporate the large interindividual variability with respect to the metabolic and cellularity characteristics of their adipose depots. This may translate into different 'thresholds' for adipocyte size and consequently different maximal capacities for a given adipocyte number. Overall, the value of this hypothesis is that it provides a basic explanation for the persistence of the energy gap driving weight regain in both static (during weight maintenance) and dynamic (during weight regain) phases of the relapse to obesity, and in addition, it frames the integration of longterm signals for stored energy and short-term signals of nutrient availability in a manner that links both to the cellular and metabolic characteristic of adipose tissues.

\section{Nutrient transporters: new players in weight regulation}

Another future avenue for research in the biology of weight regain may well centre on monocarboxylate transporters (MCTs). There is emerging evidence that MCTs, which drive lactate, pyruvate and ketone body transport across plasma membranes - and which are present in adipocytes and brain neurons (including glucose-sensitive hypothalamic neurons) - may act as fuel sensors of their metabolic environments. Indeed, several MCT isoforms and their substrates are known to modulate various components of weight homeostasis, ranging from their central effects on food intake to peripheral effects in the browning of white adipose tissue (13). In reviewing the role of MCTs as new players in weight homeostasis, Carneiro and Pellerin (14) point out that the proof of concept about the potentially critical role of MCT in weight regulation has recently been demonstrated in the ability of haploinsufficient MCT1 mice to resist obesity when exposed to an obesogenic diet - by reducing food intake and intestinal nutrient absorption, as well as increasing resting metabolic rate. Furthermore, they emphasized that the expression of cerebral MCT isoforms can be modulated by alterations of peripheral metabolism and that con- sidering their distribution and their suggested functions in various aspects of metabolism, it is likely that their expression both in the central nervous system and in the periphery might be influenced by nutritional manipulations including caloric restriction and refeeding. They underscore the possibility that MCTs might play a key role in the adaptations taking place during weight loss and upon weight regain and that the generation of transgenic mice with tissue-specific invalidation for specific MCT isoform(s) would enable the exploration in greater depth of the role of MCTs in the biology of weight regain.

\section{Exercise and weight management: beyond the 'tyranny of the average'}

Among behavioural modifications that are prescribed for facilitating slimming diet therapy and countering weight regain, exercise is certainly the one that is most commonly advocated. The efficacy of various exercise regimes for achieving greater weight loss or for long-term weight maintenance remains, however, contentious. In many cases, the degree of weight loss is much less than that theoretically predicted on the basis of the measured energy expenditure and its presumed relationship to tissue lost. Furthermore, the average weight loss attributed to exercise per se is considered to be marginal (15). However, as Blundell et al. (16) emphasize in this issue, one should not subscribe to the 'tyranny of the average' for the average could be hiding a huge individual variability in response to exercise regimens - with some achieving substantial weight losses, others only minor or no weight losses and a few others even gaining weight (albeit mostly in the form lean body mass). To explain such large variability in response to exercise, Blundell et al. (16) take us on a historical journey through the classic studies of Jean Mayer on jute mill workers in Bengal and those of Otto Edholm on British army cadets which established the fundamental relationships between energy expenditure and energy intake. Similar with these pioneers in human energy balance, they raise the question as to what extent increased physical activity results in compensatory increases in energy intake and explore the reasons as to why such compensation varies markedly from person to person. In reviewing this topic on the impact of exercise on appetite control and energy balance, they emphasize as much upon the inevitable interference of exercise on the neurohormonal episodic control of appetite as through the longer-term effects of exercise on body composition (fat mass and FFM) and their impacts on the tonic control of appetite. They conclude that as the specific actions of exercise on each physiological component will vary in strength from person to person (according to individual physiological characteristics) and with the intensity and duration of exercise, the individual responses to exercise will be highly variable and difficult to predict. In many individuals there- 
fore, exercise may not necessarily work in concert with dieting to achieve weight loss and prevent weight regain.

\section{Lessons from bariatric surgery: beyond the 'low-hanging fruits'}

By contrast, although there are concerns about its potential adverse nutritional and psychological effects, bariatric surgery has over the past decade emerged as a most efficacious approach for achieving large and sustained weight loss as well as for the treatment of obesity-associated comorbidities. This has generated intense research activities both in humans and animal models towards identifying the biological mechanisms underlying its beneficial effects with a view towards non-surgical treatment options. As underscored by Münzberg et al. (17) in this issue, these studies have shown numerous structural, functional and molecular changes in the gut, the brain and other organs as well as changes in energy metabolism, glucose homeostasis and behaviour, and these have formed the basis of various hypotheses to explain the beneficial effects of bariatric surgery. However, a major issue in many of these studies is that it is not possible to delineate the effects of hypophagia induced by the surgical stress from the merits of the bariatric intervention per se, and furthermore, the effects that seem independent of the hypocaloric state and weight loss are variable and less clear. To quote Münzberg et al. (17) in their critical review highlighting potential mechanisms contributing to the sustained change in weight regulation that allows bariatric patients and rodent models to remain at greatly reduced body weight levels, 'after going for the low-hanging fruit, it is now time to separate irrelevant changes from mechanistically relevant ones'. They pin-point important species differences (and caveats) in attributing a role for increased energy expenditure after bariatric surgery and emphasize that although a number of changes in food choice, taste functions, hedonic evaluation, motivation and self-control have been documented in both humans and rodents after surgery, their importance and relative contribution to diminished appetite has not yet been demonstrated. Furthermore, they argue that none of the major candidate mechanisms postulated in mediating surgery-induced changes from the gut and other organs to the brain, such as gut hormones and sensory neuronal pathways, have been confirmed yet. Their general conclusion is that the elucidation of mechanisms by which bariatric surgery leads to sustained relative hypophagia that allows an active defence of a new body weight level would require research efforts that should focus on interventional rather than descriptive approaches.

\section{Concluding remarks}

The main outcome of this conference pertaining to dieting in the treatment of obesity could not have been more appropriately articulated than the concluding remarks made by MacLean et al. (12), to quote, 'weight loss awakens the body's defense system in a manner that is persistent, saturated with redundancies, and well-focused on the objective of restoring the body's depleted energy reserves. Successful, long term weight loss requires recognition of the strength and persistence of these biological pressures, and a better understanding of how they may be countered with environmental, behavioral, pharmaceutical or other interventions. To be effective, interventions aimed at preventing weight regain will likely need to be as comprehensive, persistent, and redundant as the biological adaptations they are attempting to counter'. On the other hand, given the increasing prevalence of dieting to lose weight among those in the healthy normal range of body weight (because of media, family, societal and sports performance pressures) and the emerging evidence that dieting in these population groups is a strong predictor of future weight gain and cardiometabolic risks, one may also add that among strategies to control the obesity epidemic, primary preventive measures should also target these lean 'dieting-prone' population groups.

\section{Conflict of interest statement}

All authors declare no conflict of interest.

\section{References}

1. Procope J. Hippocrates on Diet and Hygiene. Zeno: London, 1952.

2. Cannon G, Einzig H. Dieting Makes You Fat. Century Publishing: London, 1983.

3. Mann T, Tomiyama AJ, Westling E, Lew AM, Samuels B, Chatman J. Medicare's search for effective obesity treatments: diets are not the answer. Am Psychol 2007; 62: 220-233.

4. Bacon L, Aphramor L. Weight science: evaluating the evidence for a paradigm shift. Nutr J 2011; 10: 9.

5. Montani JP, Schutz Y, Dulloo AG. Dieting and weight cycling as risk factors for cardiometabolic diseases: who is really at risk? Obes Rev 2015; 16 (Suppl. 1): 7-18.

6. Hill AJ. Does dieting make you fat? Br J Nutr 2004; 92(Suppl. 1): S15-S18.

7. Lowe MR. Dieting: proxy or cause of future weight gain? Obes Rev 2015; 16 (Suppl. 1): 19-24.

8. Saarni SE, Rissanen A, Sarna S, Koskenvuo M, Kaprio J. Weight cycling of athletes and subsequent weight gain in middleage. Int J Obes 2006; 30: 1639-1644.

9. Pietiläinen KH, Saarni SE, Kaprio J, Rissanen A. Does dieting make you fat? A twin study. Int J Obes 2012; 36: 456-464.

10. Dulloo AG, Jacquet J, Montani JP, Schutz Y. How dieting makes the lean fatter: from a perspective of adipostats and proteinstats awaiting discovery. Obes Rev 2015; 16 (Suppl. 1): 25-35.

11. Bosy-Westphal A, Jäger J, Lagerpusch M, Skurk T, Müller MJ. Deep body composition phenotyping during weight cycling: relevance to metabolic efficiency and metabolic risks. Obes Rev 2015; 16 (Suppl. 1): 36-44. 
12. MacLean PS, Higgins JA, Giles ED, Sherk VD, Jackman MR. The role for adipose tissue in weight regain after weight loss. Obes Rev 2015; 16 (Suppl. 1): 45-54.

13. Carrière $\mathrm{A}$, Jeanson $\mathrm{Y}$, Berger-Müller $\mathrm{S}$ et al. Browning of white adipose cells by intermediate metabolites: an adaptive mechanism to alleviate redox pressure. Diabetes 2014; 63: 32533265 .

14. Carneiro L, Pellerin L. Monocarboxylate transporters: new players in body weight regulation. Obes Rev 2015; 16 (Suppl. 1): $55-66$
15. Garrow JS. Exercise in the treatment of obesity: a marginal contribution. Int J Obes Relat Metab Disord 1995; 19(Suppl. 4): S126-S129.

16. Blundell JE, Gibbons C, Caudwell P, Finlayson G, Hopkins M. Appetite control and energy balance: impact of exercise. Obes Rev 2015; 16 (Suppl. 1): 67-76.

17. Münzberg H, Laque A, Yu S, Rezai-Zadeh K, Berthoud HR. Appetite and body weight regulation after bariatric surgery. Obes Rev 2015; 16 (Suppl. 1): 77-90. 